\title{
OPTIMIZATION LOOP ALGORITHM FOR ADSORBED NATURAL GAS STORAGE SYSTEMS
}

\author{
JOÃO S. BRASIL LIMA, BRUNO G. CHIEREGATTI ${ }^{1,2}$, ERNANI V. VOLPE ${ }^{2}$, \\ MARCELO T. HAYASHI ${ }^{2,3}$ \\ ${ }^{1}$ Mauá Institute of Technology \\ Mechanical Engineering Department \\ Praça Mauá, 01, São Caetano do Sul, SP, Brazil \\ 09580-900 \\ joao.brasil@maua.br \\ ${ }^{2}$ Research Centre for Gas Innovation (RCGI) \\ Polytechnic School of University of São Paulo \\ Av. Prof. Mello Moraes, 2231 05508-000 \\ São Paulo, SP, Brazil \\ ${ }^{3}$ Federal University of ABC \\ Center for Engineering, Modeling and Applied Social Sciences \\ Rua Frei Caneca, Vila São Pedro, Santo André, SP, Brazil \\ 09210-170
}

Key Words: Porous Media, Adjoint, Optimization, Inverse Design.

\begin{abstract}
In the past few years, the development of inverse design and optimization methods has opened up new possibilities. The so-called Adjoint method is of great significance in that context, since it permits high fidelity to flow-physics at comparatively low computational costs. The present work is a sequel of a previous one presented in WCCM2018, called "On the use of the Adjoint Method to evaluate sensitivities in adsorbed natural gas storage systems". where one have developed and validated an Adjoint based approach to computing sensitivity derivatives for adsorbed natural gas (ANG) storage systems. The main goal of this work is to, by using the approach to compute sensitivities presented before, obtain and validate a basic structure of an optimization loop algorithm (OLA) for optimization of natural gas storage systems. Both flow and Adjoint solvers, which were previously developed, are assembled in FREEFEM++ platform. The OLA consists on solving sequential problems to achieve an optimal configuration of parameters that maximize/minimize an objective functional. It starts by solving the primal problem (flow solver), which consists in a physics flow solution, followed by the dual problem, based on the Adjoint Method. With both solutions, the OLA receives the sensitivity derivatives with respect to parameters and, if the configuration is not the optimal, a new values of parameters is obtained and the cycle restarts. To validate the OLA, we make use of the inverse design optimization, defining the objective functional as the mean square error, MSE, of the actual density of adsorption distribution q, with respect to an user--defined target distribution, qt. The strategy is generated a target distribution with a known filling flow curve and the OLA, starting the optimization cycles with other flow curve, minimizing the functional,
\end{abstract}


finding the same curve as we use to generate qt. The results of the several tests showed that the OLA have the capacity to regenerate the original curves, proving the consistency of the source code. The next step for the future researchers is the application for the engineering purposes, by using operational requirements to optimize the process.

\section{INTRODUCTION}

Worldwide, the energy market is going through a phase of intense research and development efforts. In this scenario, the Natural Gas appears as a source of energy of growing relevance, owing to both its direct uses and to the possibilities it offers regarding the process of gas reform, for hydrogen production and carbon capture. The growth of its share of the energy market prompts the need to optimize the chain of production, processing, transportation and storage of Natural Gas.

Nowadays, the Transportation companies have two most common technologies to deliver NG without pipelines: Compressed Natural Gas (CNG) which consists of a tank that receives NG through a compressor and Liquified Natural GAS (LNG) which involves cryogenics procedure. The main advantage of LNG is the huge storage capacity, but the costs to refrigerate the gas and the thermal insulation demands make the technology viable only for large scale facilities.

Moreover, there is a gap between the capacity of these two technologies and the Adsorbed Natural Gas (ANG) technology becomes an attractive alternative for transport or
storage purposes. To a reasonable extent, the method provides a means of storing gas at
substantially higher concentration than can be achieved with CNG at the same pressure.
Although it does not attain the density that is typically found with LNG, it is potentially nuch
simpler, since it does not require the energy-demanding of liquefaction process [1].
The ANG requires the menagement of the heat produced by the adsorption menoma and the optimization of the filling process is necessary to use the technology potential. Under

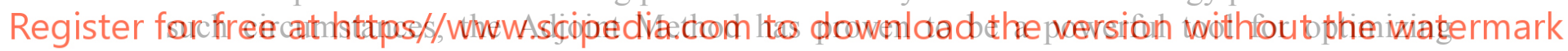
complex systems, where a high fidelity representation of the physics is essential. It has shown to be particularly suitable to tackle problems with large numbers of control parameters, and several possibilities of optimum criteria. For it only requires two converged solutions to compute sensitivity gradients, regardless of their dimensionality and for any particular measure of merit.

\section{FLOW AND ADJOINT EQUATIONS}

As commented before, in the previous paper [6] both flow and adjoint equations were derived and presented, with the respective boundary conditions. For convenience, they are, once again, shown below. The assumptions and hypothesis are fully explained in the aforementioned reference. First, the flow equations:

$$
\begin{aligned}
& \epsilon_{t}\left(\frac{1}{T} \cdot \frac{\partial p}{\partial t}+\frac{p}{T^{2}} \cdot \frac{\partial T}{\partial t}\right)+\rho_{b} \frac{\partial q}{\partial t}+\nabla \cdot \vec{G}=0 \\
& \vec{G}+N_{p} \rho_{g} \nabla p=0 \\
& C_{e f f} \frac{\partial T}{\partial t}-\epsilon_{t}\left(\frac{\gamma-1}{\gamma}\right) \frac{\partial p}{\partial t}+\nabla \cdot(\vec{G} \cdot T)-\frac{1}{P e} \nabla^{2} T-\frac{\rho_{\mathrm{b}} \Delta H}{M_{g}} \cdot \frac{\partial q}{\partial t}=0
\end{aligned}
$$




$$
q=\rho_{a d s} \cdot W_{0} \cdot \exp \left[-\left(\frac{A}{\beta \mathrm{E}_{0}}\right)^{n}\right]
$$

Eq.(1) is the continuity equation, eq.(2) is the momentum equation, eq. (3) is the Energy equation and eq. (4) is the Dubinin-Astakov (DA) model $[4,5]$. The variables that appear in the equations are:

- $\epsilon_{t}$ : total porosity of adsorbent bed (no-dimensional);

- $\rho_{b}$ : density of adsorbent bed;

- $q$ : density of adsorption;

- $\vec{G}=\rho_{g} \vec{u}$ : specific mass flux vector

- $\rho_{g}$ : free gas density

- $N_{p}=\frac{K \rho_{\infty}}{\mu l_{r e f} v_{\infty}}:$ pressure number

- $\mu$ : gas viscosity;

- $K$ : permeability of the adsorbent bed;

- $C_{e f f}=\left(\epsilon_{t} \rho_{g}+\rho_{b} q\right) C_{p g}+\rho_{b} C_{p s}$, which $C_{p g}$ and $C_{p s}$ represents the specifics heat of gas and adsorbent respectively;

- $P_{e}=\frac{\rho_{\infty} C_{p g} v_{\infty} l_{\text {ref }}}{\lambda_{\text {ref }}}$ : Péclet number;

- $\lambda_{\text {eff }}=\epsilon_{t} \cdot \lambda_{g}+\left(1-\epsilon_{t}\right) \lambda_{s}$ : effective thermal conductivity

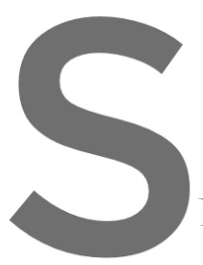

- $\rho_{\text {ads }}$ adsorbe

- $A=R T \ln$

Now, the adjoint equations developed in the re

$\left\langle\delta \rho, \Gamma_{11} \partial_{0} \sigma+\Gamma_{12} \partial_{0} \theta+\Gamma_{13} \nabla \sigma+\Gamma_{14} \nabla \cdot \vec{\Psi}+\right.$
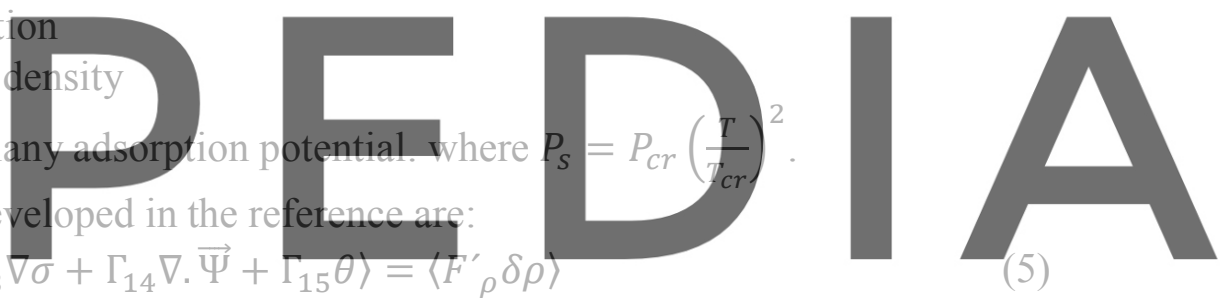

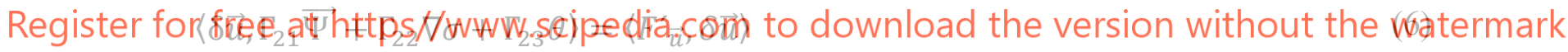

$$
\left\langle\delta \mathrm{T}, \Gamma_{31} \partial_{0} \sigma+\Gamma_{32} \partial_{0} \theta+\Gamma_{33} \nabla \theta+\Gamma_{34} \nabla \cdot \vec{\Psi}+\Gamma_{35} \theta+\Gamma_{36} \nabla^{2} \theta\right\rangle=\left\langle F_{T}^{\prime} \delta T\right\rangle
$$

where $\sigma, \theta, \Psi$ are lagrange multipliers and, hence, the adjoint variables and $\Gamma_{i i}$ are numerical coefficients that depend only of fluid and geometry parameters and are presented in the previous paper [6]. Both systems of equations were were implemented in FreeFEM++ platform, a high level system that solves partial differential equations [2]. In the figure 1 the geometry of the tank is presented as well as the numerical mesh:
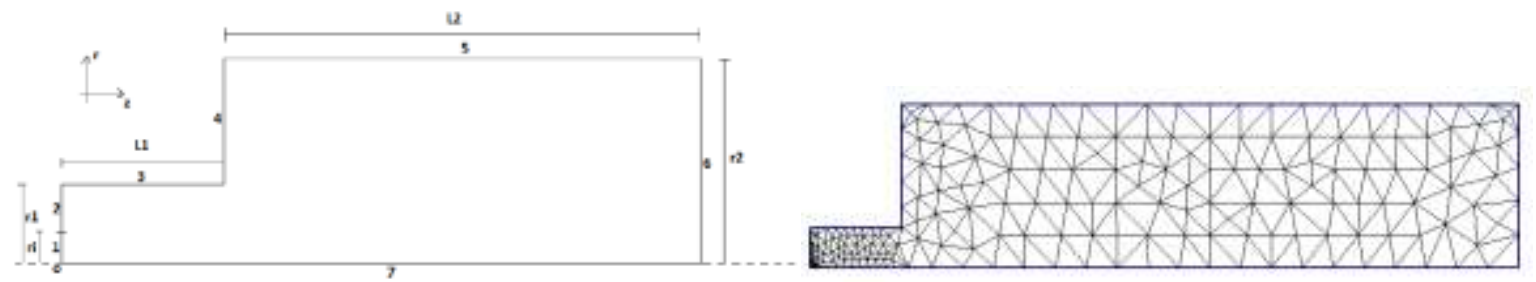

Figure 1. Left: Schematic of a 2D axi-symmetric ANG Storage System "o": origin of coordinate system. Right: Numerical mesh

The boundary conditions ( $\left.\mathrm{BC}^{\prime} \mathrm{s}\right)[6]$ for both problems are presented in the table 1: 
Table 1: Boundary conditions for the dual problem

\begin{tabular}{|c|c|c|c|}
\hline BC & Flow & Flow Linearized & Adjoint \\
\hline Inlet & $\begin{array}{l}\rho \vec{u}- \\
f\left(X, t, G_{m}\right)=0 \\
T-T_{i n}=0\end{array}$ & $\begin{array}{c}(\delta \rho \vec{u}+\rho \delta \vec{u})-\frac{\partial f}{\partial G_{m}} \delta G_{m}-\frac{\partial f}{\partial t_{a c}} \delta t_{a c} \\
=0 \\
\delta T-\delta T_{i n}=0\end{array}$ & $\begin{array}{c}\vec{\Psi} \cdot \vec{n}=0 \\
\theta=0\end{array}$ \\
\hline Outflow & $P-P_{\text {out }}=0$ & $(\delta \rho \mathrm{T}+\rho \delta \mathrm{T})-\delta P_{\text {out }}=0$ & $\begin{array}{l}\quad \sigma=0 \\
\nabla \theta \cdot \vec{n} \\
=-(P e \rho \vec{u} \cdot \vec{n}) \theta\end{array}$ \\
\hline Wall & $\begin{array}{l}\rho \vec{u}=0 \\
-\nabla T \cdot \vec{n}=\mathrm{Nu}(T- \\
\left.T_{\text {ext }}\right)\end{array}$ & $\begin{array}{l}(\delta \rho \vec{u}+\rho \delta \vec{u})=0 \\
-\nabla \delta T \cdot \vec{n}=\delta \mathrm{Nu}\left(T-T_{\text {ext }}\right)+N u \delta T- \\
N_{\text {ext }}=0\end{array}$ & $\begin{array}{c}\vec{\Psi} \cdot \vec{n}=0 \\
\nabla \theta \cdot \vec{n}=\mathrm{Nu} \theta\end{array}$ \\
\hline Sym. & $\begin{array}{l}\rho \vec{u} \cdot \vec{n}=0 \\
\nabla T \cdot \vec{n}=0\end{array}$ & $\begin{array}{l}(\delta \rho \vec{u}+\rho \delta \vec{u}) \vec{n}=0 \\
\nabla \delta T \cdot \vec{n}=0\end{array}$ & $\begin{array}{l}\vec{\Psi} \cdot \vec{n}=0 \\
\nabla \theta \cdot \vec{n}=0\end{array}$ \\
\hline
\end{tabular}

where $f\left(X, t, G_{m}\right)$ is the filling flow function, in terms of the inlet geometry $(X)$, time $(t)$ and average mass flux (Gm); $T_{\text {ext }}$ is the ambient temperature and $N_{u}$ is the Nusselt number defined
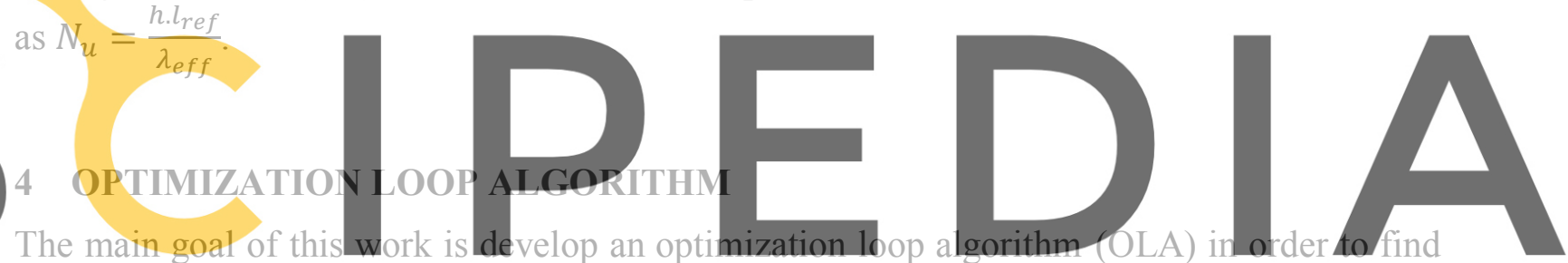

optimal operation configurations that maximizes/minimizes and objective functional. The loop

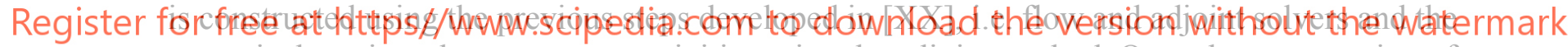
numerical routines that compute sensivities using the adjoint method. Once the computation of sensitivities was validated in the previous work, one can now proceed to the construction of the OLA.

\subsection{Structure of an optimization loop}

The OLA proposed has the following structure: 


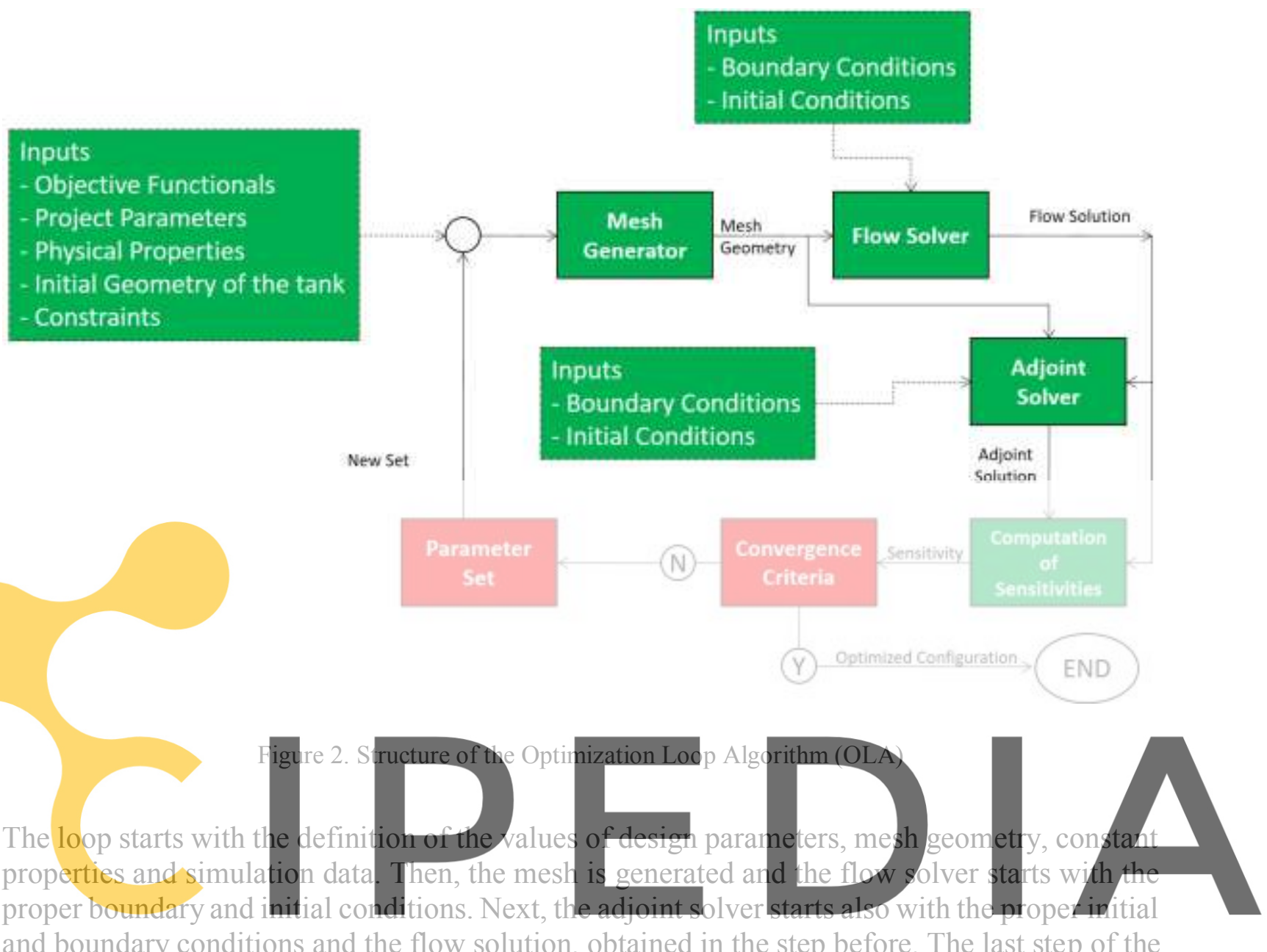

and boundary conditions and the flow solution, obtained in the step before. The last step of the

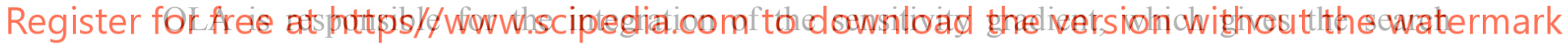
direction of all design parameters. With this direction, the values of the parameters are changed using a steepest decent method. If the configuration of parameters is optimal, the loop stops, if it is not, a new cycle starts and the OLA keeps iterating until the convergence is achived.

\subsection{Validation of the OLA}

With the programming of the \$OLA $\$$, validation tests were performed to test the integration of the whole source codes. We choose the inflow boundary condition as the test, changing the parametrization of the flow curve:

$$
f\left(G_{z}, G_{r}\right)=\left(2 \cdot \eta G_{m} \cdot\left(1-\frac{r^{2}}{r_{i}^{2}}\right), 0\right)
$$


In the eq. (8) $\boldsymbol{G}_{\boldsymbol{m}}$ was considered constant and $\boldsymbol{\eta}$ represents a function, described by the design parameters and dependent on time. In the other words, $\boldsymbol{\eta}$ is a factor that multiplies the nominal average mass flux $\boldsymbol{G}_{\boldsymbol{m}}$. The objective function was determined by considering the monitoring of the \$OLA\$ answer. The idea is the inverse design application, where we use a known distribution of the density of adsorption inside the tank, that we call target. Then, we impose an volumetric $\boldsymbol{V}_{\boldsymbol{t}}$ average quadratic error between the currently distribution and the target as measure of merit, presented in the eq. (9):

$$
R=\frac{1}{T \cdot V_{t}} \int_{0}^{T} \oint_{D} \frac{\left(q-q_{t}\right)^{2}}{2} d V d t
$$

Where $q_{t}$ is the known distribution (target) and $\boldsymbol{q}$ is the density of adsorption distribution evaluated in the optimization cycle. By evaluating the variation of objective function, $\partial \boldsymbol{R}$, it yields the eq. (10):

$$
R=\frac{1}{T \cdot V_{t}} \int_{0}^{T} \oint_{D}\left(q-q_{t}\right)^{2} \delta q d V d t
$$

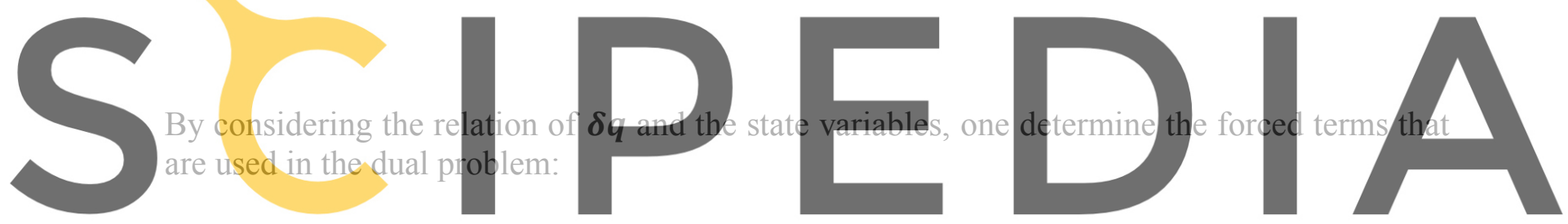

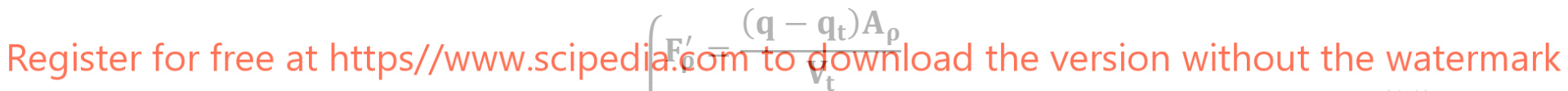

$$
\left\{\begin{array}{c}
\mathbb{F}_{\mathrm{u}^{\mathrm{i}}}^{\prime}=0 \\
\mathbf{F}_{\mathbf{T}}^{\prime}=\frac{\left(\mathbf{q}-\mathbf{q}_{\mathbf{t}}\right) \mathbf{A}_{\mathbf{T}}}{\mathbf{V}_{\mathbf{t}}}
\end{array}\right.
$$

For the following tests, the mesh geometry, and the simulation setup are the same as those of the primal and dual problem validations which are presented in the previous work [6]. One use the same value of the volumetric flow rate that was used in the dual problem validation [6]:15 $\mathrm{L} / \mathrm{min}$ which represents $\boldsymbol{G}_{\boldsymbol{m}}=5.5615 \mathrm{~kg} / \mathrm{m}^{2} \mathrm{~s}$. The curve of the inflow mass flow will be parameterized with the Bernstein polynomials [7]. Originally developed to describe geometrical shapes, the definition of these polynomials are presented in eq.(12):

$$
\mathrm{S}_{\mathrm{n}}=\sum_{\mathrm{k}=0}^{\mathrm{n}} b_{k} C_{n, k} x^{k}(1-x)^{n-k}
$$


Where $\boldsymbol{b}_{\boldsymbol{k}}$ represent the actual control parameters, which are evaluated by means of the Adjoint method. The variable $\boldsymbol{x}$ is limited to values between 0 and 1 . This is easily programmed in the OLA defining $\boldsymbol{x}=\boldsymbol{t} /$ totaltime. The coefficients $\boldsymbol{C}_{\boldsymbol{n}, \boldsymbol{k}}$ are Newton binomial coefficients. The validation tests were made with two different polynomials, with degree 2 and 5 where the control parameters are the coefficients of the polynomials. In the first test, the target distribution is $\boldsymbol{\eta}=\mathbf{- 4} \boldsymbol{t}^{\mathbf{2}}+\mathbf{4 t}$ and the total filling time is 120s. The OLA started with $\boldsymbol{b}_{1}=\mathbf{1 . 2}$ and $\boldsymbol{b}_{2}=$ 1. 0 and the results of the optimization are presented in the fig. 3 :
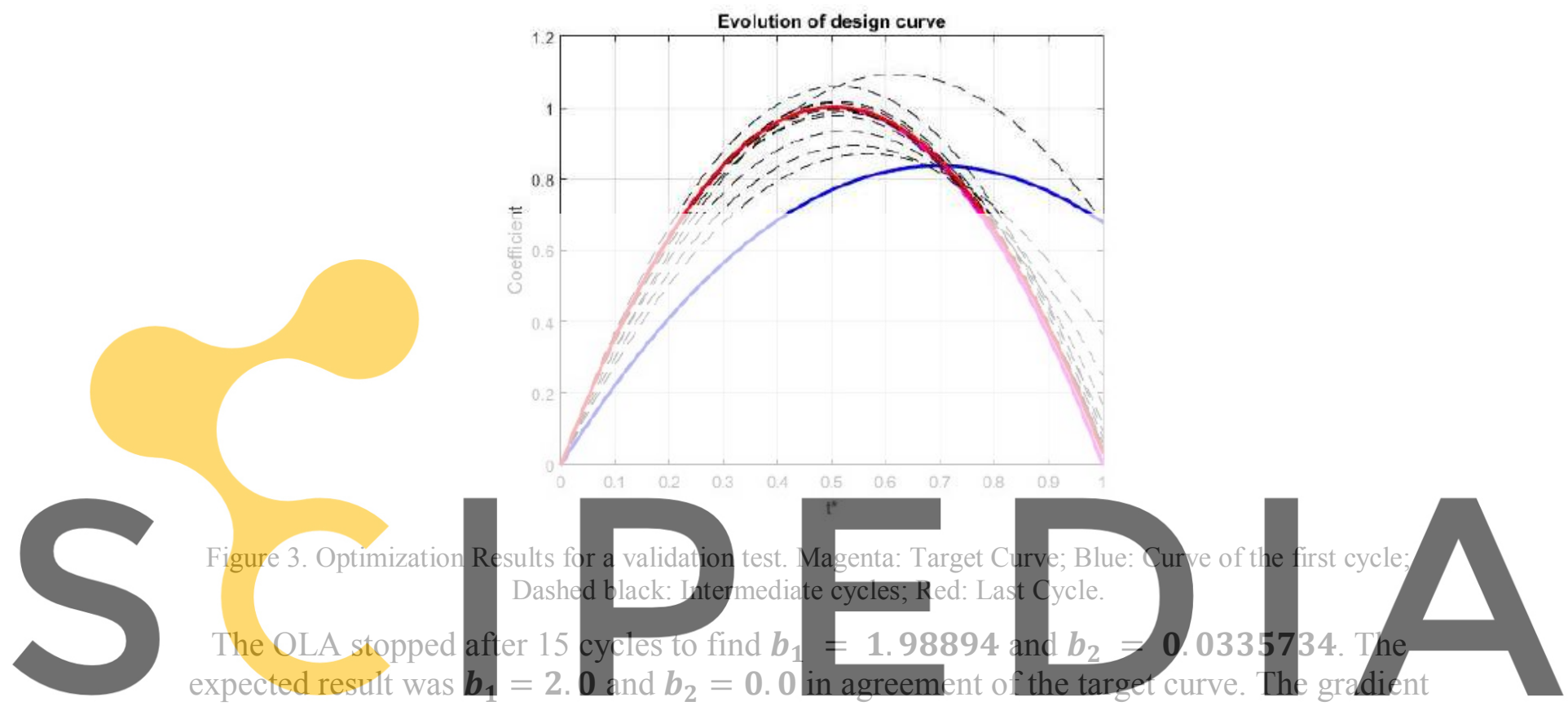

magnitude getting close to $\mathbf{1 0}^{-7}$ for both design parameters. Next, one presents, in fig. 4 , the

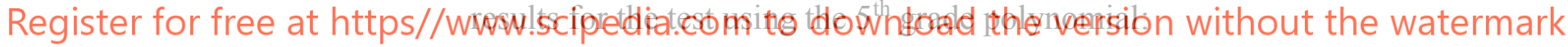

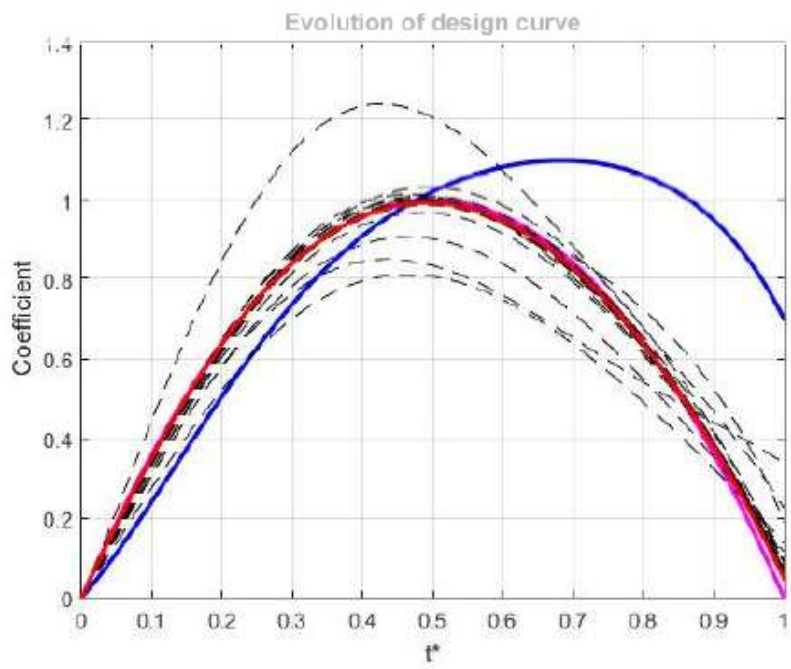

Figure 4. Optimization Results for a validation test with 5th degree Bernstein Polynomials. Magenta: Target Curve; Blue: Curve of the first cycle; Dashed black: Intermediate cycles; Red: Last Cycle. 
The OLA starts with $\boldsymbol{b}_{\mathbf{1}}=\mathbf{0 . 4}, \boldsymbol{b}_{\mathbf{2}}=\mathbf{1 . 4}, \boldsymbol{b}_{\mathbf{3}}=\mathbf{0 . 9}, \boldsymbol{b}_{\mathbf{4}}=\mathbf{1 . 4}$ and $\boldsymbol{b}_{\mathbf{5}}=\mathbf{0 . 7}$. After 35 cycles, the OLA stoped, with the values changed to $b_{1}=0.762011, b_{2}=1.28003, b_{3}=$ 1.12153, $b_{4}=0.781715$ and $b_{5}=0.0475444$ and the objective function coming to $\boldsymbol{R}=2.82846 .10^{-8}$. The recovering of the target curve were suitable, regardless of the difference in degree between the target and the current parametrization. The sensitivity gradient component achieved values between $\mathbf{1 0}^{-\mathbf{8}}$ and $\mathbf{1 0}^{-9}$. The validation tests presented the OLA capacity to change the filling flow curves to achieve the extreme of a given objective function [9].

\section{OPTIMIZATION APPLICATION}

After the validation of the OLA, it can be used to tackle optimization problems. Now, here is presented an application that can be used in storage facilities. As the adsorption is an exothermal phenomenon, the effect that maximum adsorption capacity is attained under isothermal conditions. Whence comes the need for heat transfer, to counteract the exothermic character the adsorption process exhibits. On the other hand, adsorption rates also depend on the thermodynamic state, i.e. $(P, T)$ distributions. It is under such conditions that the search for an optimum filling curve must take place.

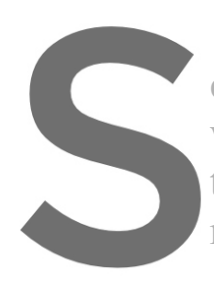

An attempt is made

while pressure is linearl

thus obtained, are then

resulting distribution
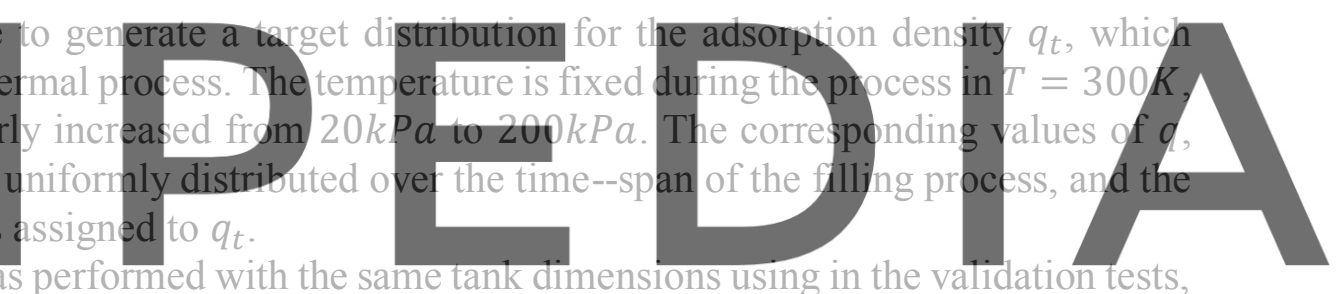

the initial pressure and temperature setted in $20 \mathrm{kPa}$ and $300 \mathrm{~K}$ respectively and the time of

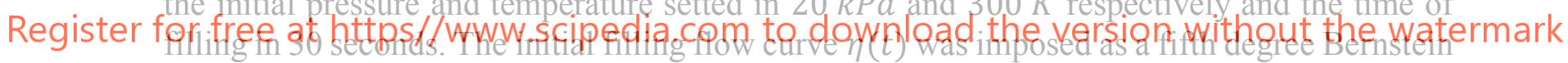
polynomial with following values: $b_{0}=0.0, b_{1}=1.0, b_{2}=1.0, b_{3}=1.0, b_{4}=1.0$, $b_{5}=1.0$. The average mass flux was $G_{m}=11.123 \mathrm{~kg} / \mathrm{m}^{2} s$ which corresponds a volumetric flow of the 30 LPM. Below, in fig. 5, the results are shown: 


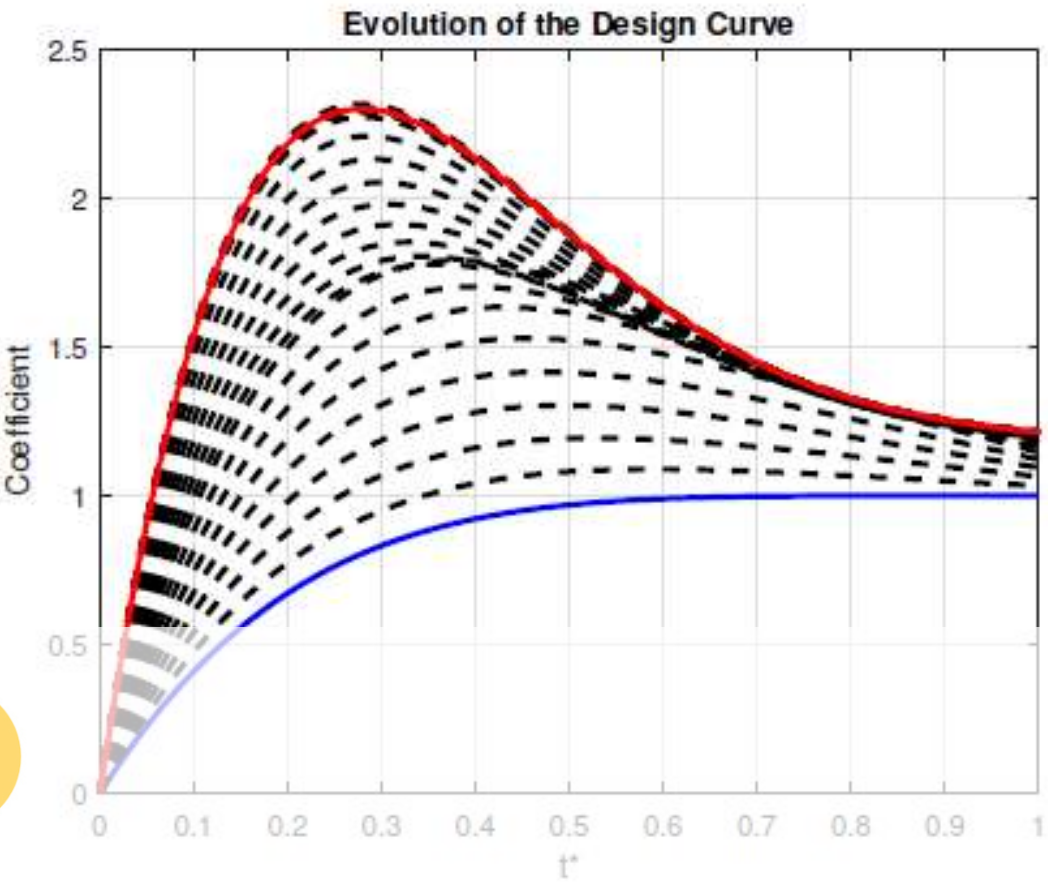

Figure 5. Evolution of the sensitivity gradient during the optimization

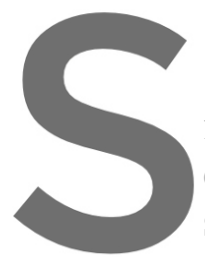

\section{After 19 cycles, the} function was 8.8.10of the function $\eta(t)$ is systematic optimization

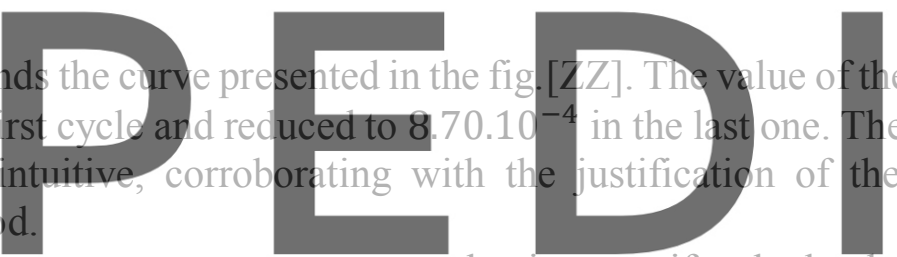

The similarity of the design curves prompts a new test, that is, to verify whether by changing

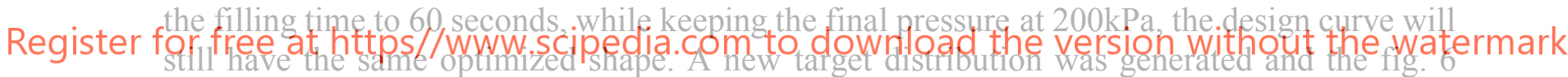
presents the evolution of the design curves. 


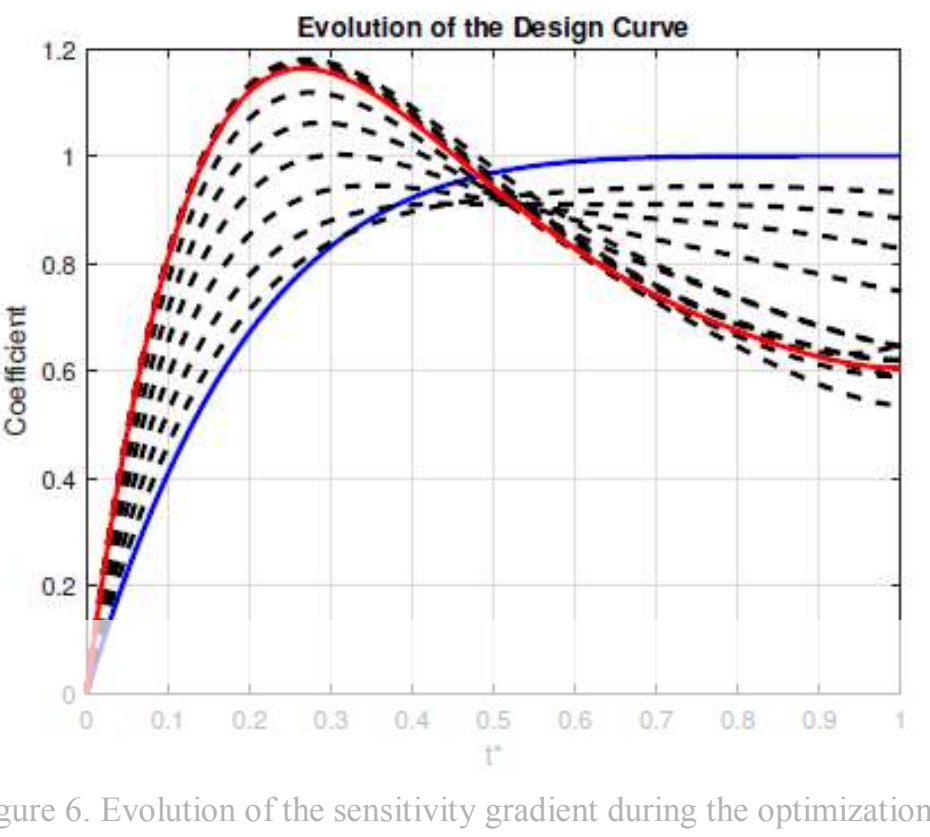

After only 14 cycles, the objective function reduces from $2.86 \cdot 10^{-3}$ to $2.86 \cdot 10^{-4}$. The initial solution was alre measure of merit. The $0.585112, b_{3}=0.90412, b$

6 CONCLUSIONS
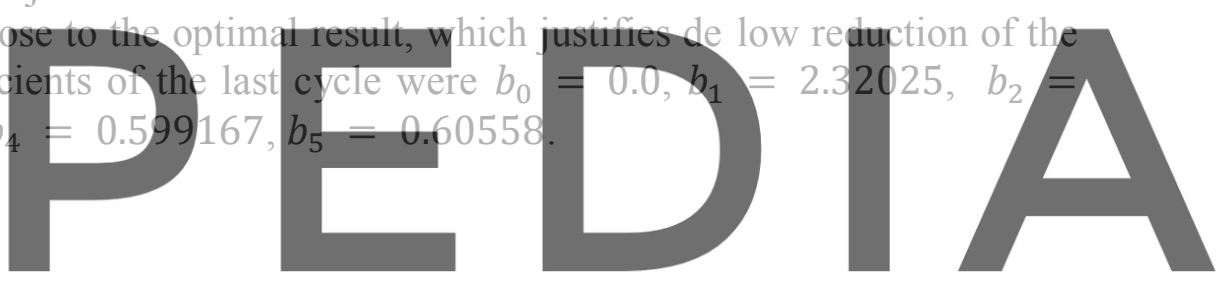

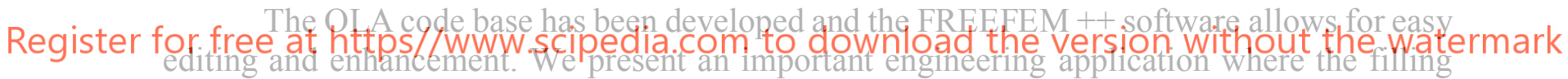

flow curve was controlled based on the desired density of adsorption distribution. The nonintuitive result shows that we not be able to find this result without a systematic analysis made by the OLA.

However, we need some improvement, as the temperature is not controlled by this curve, being required a heat transfer parameters, as Nusselt Number, inflow and external temperatures. We hope to present this evolution in the next congress.

Also, the geometric sensitivities gradient and the modification of equations, considering two or more gases are other steps of the research, where we can also combine this parameters to optimize active heat control management devices, such as heat fins and internal heat exchangers, as seen in publications by the research group [ARTIGO COBEM].

One more continuity of the research is the changing of adsorbed materials and the tests using carbon dioxide $\left(\mathrm{CO}_{2}\right)$, entering in a important subject related do sustainability. A preliminary study show that Zeolitic Imidazolate Framework (ZIF-8) materials has a great potencial of a $\mathrm{CO}_{2}$ adsorption. The OLA is able to maximize this process and contribute por a $\mathrm{CO}_{2}$ separation devices [8]. 


\section{REFERENCES}

[1] JUDD, R. W.; GLADDDING, R. E. A. The Use of Adsorbed Natural Gas Technology for Large Scale Storage. BG Technology, Gas Research and Technology, Gas Research and Technology Centre, 1998

[2] HECHT, F.; New development in FreeFem++. Journal of Numerical Mathematics, 2012, no 3-4, 251-265

[3] JUDD, R. W; GLADDDING, D.T.M; HODRIEN, R.C; BATES, D.R; INGRAM, J.P;ALLEN, M. The use of adsorbed natural gas technology for large-scale storage. BG Technology, Gas research and Technology Centre., 1998.

[4] SAHOO, P.K; JOHN, M; NEWALKAR,B.L; CHOUDHARY,N.V; AYAPPA,K.G; Filling characteristics for an activated carbon based adsorbed natural gas storage system. I \& EC research, 2011.

[5] MOTA, J; SAATDJIAN,E; TONDEUR,D; A simulation model of high capacity methane adsorptive storage system. Adsorption 1, 1995.

[6] CHIEREGATTI, B.G; BRASIL LIMA, J.S.; VOLPE, E.V; HAYASHI, M.T; On the use of the Adjoint Method to Evaluate Sensitivities in Adsorbed Natural Gas Storage Systems. 13th World Congress on Computational Mechanics (WCCM XIII), New York City, 2018.

[7] KULFAN, B.; BUSSOLETTI, J. Fundamental parametric geometry representations for aircraft component shapes. 11th Multidisciplinary Analysis and Optimization Conference, 2006.

[8] BOSE, R. Ethiraj, J et al. Adsorption of hydrogen and carbon dioxide in zeolitic imidazolate framework structure with SOD topology: experimental and modelling studies. Journal of the Adsorption Society (2020)

[9] CHIEREGATTI, B. G., Optimization based on the Adjoint Method for Adsorbed NaturalGas Storage Systems, Ph.D. thesis, University of S ao Paulo, S ao Paulo, Brazil, 2019. 